\title{
Intrinsic Rhythmic Fluctuation of Membrane Voltage Evoked by Membrane Noise in the Hodgkin-Huxley System
}

\author{
S. KUANG ${ }^{a, b}$, J. WANG ${ }^{a}$ AND T. ZENG ${ }^{a, c, *}$ \\ ${ }^{a}$ School of Science, Wuhan University of Technology, Wuhan 430070, China \\ ${ }^{b}$ German Primate Center, Kellnerweg 4, 37077 Goettingen, Germany \\ ${ }^{c}$ Journal Editorial Department, Jianghan University, Wuhan 430056, China
}

(Received August 5, 2009)

\begin{abstract}
We study the influence of membrane noise on the fluctuations of membrane voltage in the Hodgkin-Huxley neuronal system for the giant axon of squids. We find that the resting potentials of membrane patches in the absence of any external stimulus, exhibit apparent rhythmic fluctuation behaviors. The intrinsic fluctuation frequencies of membrane voltage, due to channel noise, thermal noise, or both, roughly share the same frequency ranges, despite that the fluctuations arisen from the former noise are overwhelmingly larger in amplitude than that from the latter. We also show that the rhythmic fluctuations can help enhance the encoding of weak signals within the rhythmic frequencies ranges and thus contribute positively to the information processing in the nervous system.
\end{abstract}

PACS numbers: 05.45.-a, 87.10.-e

\section{Introduction}

Signal processing in the nervous system always occurs in the presence of external perturbations like random background synaptic activity and internal fluctuations of membrane noise. Recently, understanding the neuronal basis of information processing, especially in a noisy environment has received considerable attention [1-4]. Stochasticity and fluctuations in the membrane of nervous system in general are of great interest for at least three reasons.

First, fluctuations and noise may play a constructive and essential role in the function of the nervous system through the phenomena of stochastic resonance [5-7]. It has been suggested that stochastic ion channel fluctuations are the noise source associated with stochastic resonance effects in sensory systems [8-10].

Second, fluctuations and noise will likely place limits on the accuracy of signal processing carried out by the nervous system, such as the coincidence detection computations which are believed to be performed in the auditory system [11-14].

Third, uncertainties and noise are inherent in the neuronal system and it seems likely that the encoding of information in the nervous system has been naturally designed to minimize the effects of fluctuations and noise. It has been illustrated that ion channel stochasticity may be critical in determining the reliability and precision of spike timing [15]. Besides, recent experimental evidence

* corresponding author; e-mail: flora198234@yahoo.com.cn
[16] suggests that channel noisy current may be a source of rhythmic behaviors in the neuronal system.

Here, we attempt to provide a straightforward link between the neuronal rhythms behaviors and the membrane noise, and try to find the possible functional roles played by the rhythmic behaviors in information processing in the nervous system.

Based on the Hodgkin-Huxley (HH) neuron model for the squid giant axon [17], we investigate the influence of membrane noise on the dynamic behaviors of membrane voltage. An effective approach to evaluate or observe the intrinsic oscillation behaviors is to examine the fluctuation behaviors of membrane voltage. The membrane noise considered in this study includes ion channel gating stochasticity (also known as channel noise) [15, 18] and thermal fluctuations of ionic conductances, also referred to as the Johnson noise or thermal noise [19]. We find that the fluctuation amplitude which arises from the thermal noise is far smaller compared with that from the channel noise. However, the rhythmic oscillation behaviors always occur in the membrane voltage fluctuations in the presence of these two kinds of membrane noise. We are also interested in investigating how these fluctuation rhythms can contribute to the neural information processing of weak signals.

\section{Model description}

Ion channel gating stochasticity are caused by the random opening and closing of channels. In the stochastic version of the $\mathrm{HH}$ model the channel gating dynamics is described as a probabilistic Markov process, so in 
contrast to the deterministic model the number of open channels and hence also the membrane voltage will now exhibit abrupt fluctuations with time. The probabilities (per unit time) for a closed channel to open and for an open channel to close, are just the $\alpha$ and $\beta$ transition rate functions as those in the deterministic HH model [17]. Several computational algorithms for the simulation of stochastic ion channels have been developed and implemented [20-23] and Mino et al. [24] have recently compared the accuracy and computational efficiency of the different methods and have concluded that the one proposed by Chow and White [22] is to be preferred, which is the method we used in this study. Another source of membrane noise considered here is thermal noise of electric conductors resulting from random thermal agitation associated with the electric charges in the conductor. According to the basic Ohm theorem in circuit theory, thermal fluctuations can be viewed as either a voltage noise generator in series with the conductance, or a current noise generator in parallel. For the frequencies of interest to us here the power spectra of the thermal noise are white, i.e., independent of frequency. The output of the current noise generator has an rms value [25]: $I_{\mathrm{rms}}=\sqrt{4 K_{\mathrm{B}} T G \Delta f}$, where $K_{\mathrm{B}}$ is Boltzmann's constant, $T$ is the absolute temperature, $G$ is the conductance and $\Delta f$ is the band width. For comparison in this study all the simulation results are obtained at the experimental temperature $6.3^{\circ} \mathrm{C}$. The forward Euler method is applied to solve the stochastic differential equations.

\section{Results}

We begin our investigation by showing the time course of membrane resting potential dynamical behaviors in a membrane area of $100 \mu \mathrm{m}^{2}$ in the absence of any external stimulus, as displayed in Fig. 1. One can see occasional spikes (spontaneous action potentials) in the output of $\mathrm{HH}$ system with stochastic ion channels (see Fig. 1a), whereas the membrane voltage fluctuates slightly around the resting potential (see Fig. 1b) when only the thermal noise is considered (note the difference in the scales of the membrane voltage). Our results suggest that the fluctuations of membrane voltage evoked by ion channel gating noise is greatly larger in magnitude than that from thermal noise (see Fig. 1c). Actually, this is true in a variety of membrane sizes (data not shown here). To give a straightforward comparison between the two, we calculate the power spectral density (PSD) of voltage fluctuation and show in Fig. 1d the PSD in logarithmic scale. We can see that the stochastic channel fluctuations are roughly a factor of $10^{3}-10^{4}$ larger than the thermal fluctuations. Our calculations agree quantitatively with those of Manwani and Koch's theoretical predictions from a cortical pyramidal cell [19].

We have also analyzed the power spectral density of voltage fluctuation in normal scale. We find that there are main peaks in the PSD of voltage fluctuations when the channel noise (Fig. 2a), thermal noise (Fig. 2b) or
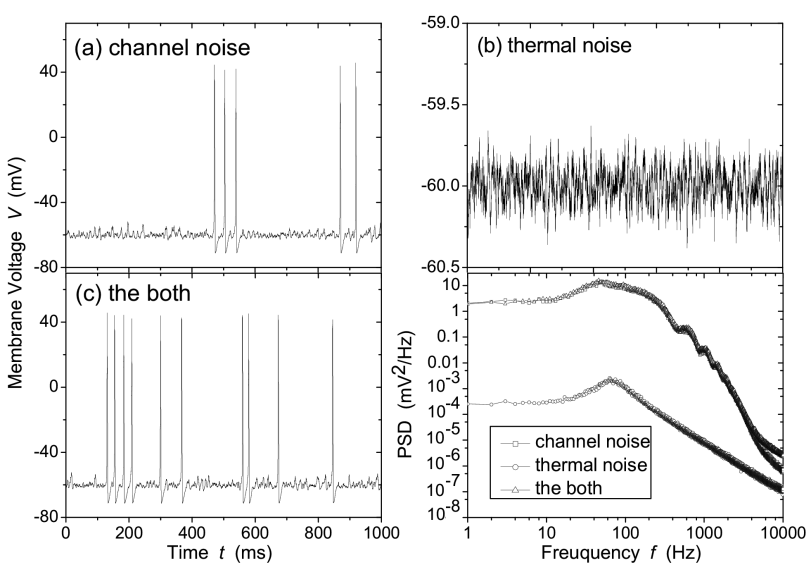

Fig. 1. Examples of membrane voltage fluctuations in the absence of any external stimulus when a membrane patch of $100 \mu \mathrm{m}^{2}$ is concerned. (a) The result in time domain for the case that only the ion channel gating stochasticity is considered. (b) The result in time domain for the case that only the thermal noise is considered. (c) The result in time domain for the case that both the two kinds of membrane noise are considered. Let us note that the membrane voltages in (b) is shown in the different scale. (d) Ensemble averaged power spectral density (PSD) of the membrane voltage fluctuations in logarithmic scale corresponding to the cases for channel noise $(\square)$, and thermal noise ( $\circ$, bottom curve) and the both $(\Delta)$.

both of them (Fig. 2c) are considered in an excitable membrane area of $100 \mu \mathrm{m}^{2}$. The appearances of peaks in the PSD may indicate the intrinsic rhythmic oscillations of membrane voltage, evoked by the biophysical realistic membrane noise. The results also reveal that the rhythmic frequency induced by channel noise is around $50 \mathrm{~Hz}$ while that by thermal noise is around $70 \mathrm{~Hz}$. This discrepancy in frequency ranges may result from the difference in the noise magnitude imposed on the HH system, for the noise intensity/influence from the channel noise is overwhelmingly larger than that from the thermal noise, as concluded from the above results. In the limitation of zero noise intensity, the intrinsic rhythmic frequencies due to thermal noise may be a more close reflection of HH system.

Next, we focus the attention on the question how the size of membrane patches influence the rhythmic fluctuation frequency of the $\mathrm{HH}$ system. We compute the peak frequencies in the PSD as a function of membrane size, as shown in Fig. 3. We can see that the intrinsic frequencies induced by the thermal noise are about $60-70 \mathrm{~Hz}$, while those arisen from the ion channel gating stochasticity are about $45-55 \mathrm{~Hz}$, in a variety of membrane sizes. Let us note that the rhythmic fluctuation frequencies from the channel noise tend to roughly increase with the increase of membrane patch sizes, but remain much smaller than those from the thermal noise. Since the noise intensity is determined by the size of the ion channel clusters in the HH system with a typical ion channel densities [19, 22], 


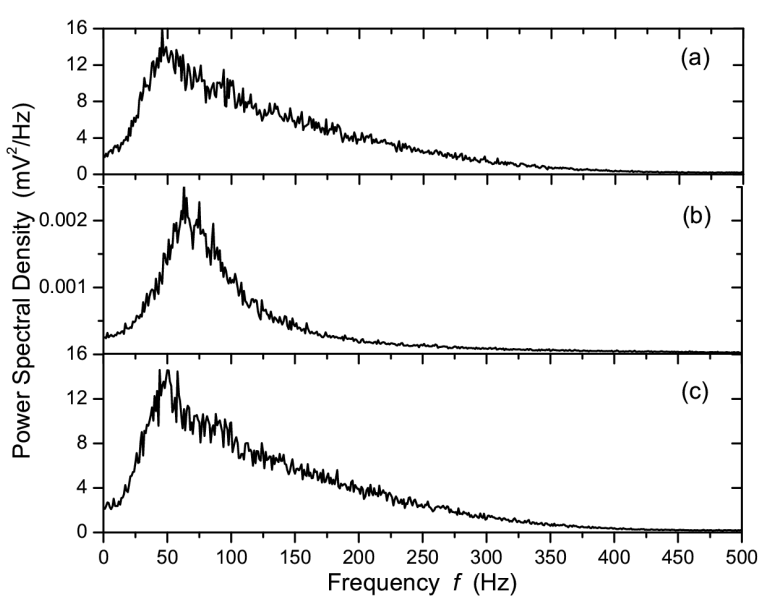

Fig. 2. Ensemble averaged power spectral density (PSD) of the membrane voltage fluctuations in a membrane patch size of $100 \mu \mathrm{m}^{2}$ in the absence of any external stimulus. (a) Only the channel noise is considered. (b) Only the thermal noise is considered. (c) Both kinds of membrane noise are considered.

the noise intensity decreases monotonously with increasing membrane size. For a large, macroscopic number of ion channels the noise intensity becomes negligible and the intrinsic frequencies may be the real reflection of the system.

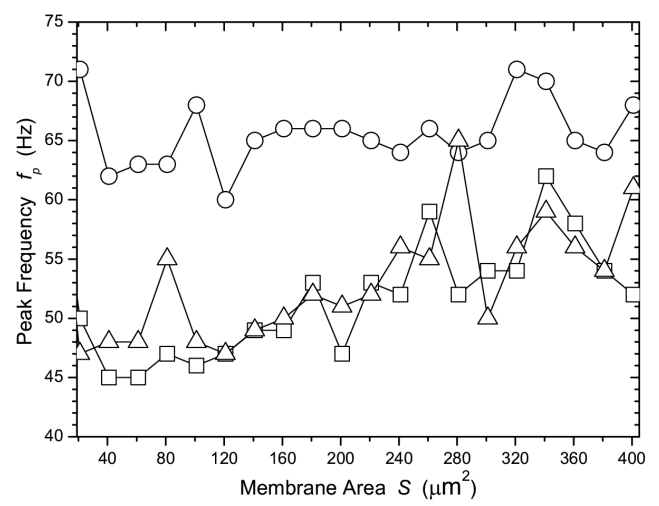

Fig. 3. The dependence of the main peak frequencies in the PSD of membrane voltage fluctuations on the size of membrane patches, for the case of channel noise $(\square)$, thermal noise $(\circ)$ and the both $(\Delta)$.

In the following we seek to gain an understanding about what functional roles do these intrinsic rhythmic oscillations may play in the enhancement of neural information processing or transmission of external signals. Since the thermal noise in a membrane patch with general sizes is extremely marginal, we will ignore the roles of thermal noise alone in the information processing of the nervous system. We calculate the output signal-to-noise (SNR) of the HH system, under channel noise or both of the two kinds of membrane noise in conjunction with a sub-threshold sinusoidal signal (injected cur- rent) for variable sizes of membrane patches, as shown in Fig. 4a and b, respectively. One can find, for both cases, that the SNR firstly increases, reaches the peak at about $70 \mathrm{~Hz}$, and then decreases with increasing signal frequencies. This dependence of SNR on the signal frequency can apply to a variety of membrane areas, $S=50,100,200 \mu \mathrm{m}^{2}$. The results suggest that the most efficient encoding of sub-threshold signal occurs at about $70 \mathrm{~Hz}$, where the $\mathrm{HH}$ system shows its intrinsic rhythmic oscillations. It is important to note that no external noise is necessary for this effect to occur, also known as intrinsic stochastic resonance, or size resonance $[8,9]$. Our results have shown that internal membrane noise can enhance a sub-threshold stimulus at its intrinsic frequency ranges.

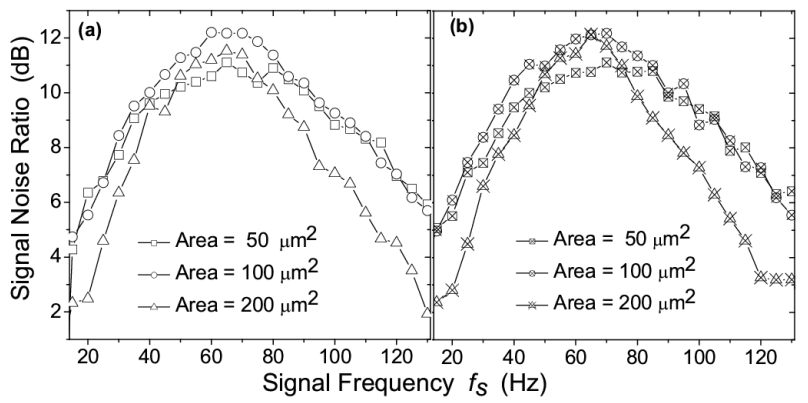

Fig. 4. The signal-noise ratio of output $\mathrm{HH}$ neuron as a function of external signal frequencies in a variety of membrane patch sizes. The results are shown for the cases of stochastic channel noise (a) and both the two kinds of membrane noise (b).

\section{Conclusions}

In summary, we have studied the intrinsic rhythmic oscillations in the presence of membrane noise in the $\mathrm{HH}$ system. Our findings show that the fluctuation magnitude arisen from the channel noise is overwhelmingly larger than that from the thermal noise. This observation is quantitatively consistent with Manwani and Koch's analytical results for a cortical pyramidal cell. In addition, we find that the membrane noise could provoke the intrinsic rhythmic oscillations in the membrane voltage fluctuation behaviors. By calculating the SNR of $\mathrm{HH}$ system we reveal that these intrinsic rhythmic behaviors, evoked by the membrane realistic noise, can help to enhance the decoding of weak signal and contribute positively to the neural information processing via the mechanism of stochastic resonance.

\section{Acknowledgments}

The authors would like to acknowledge the financial support from the Key Project of Chinese Ministry of Education (grant No. 106115). 


\section{References}

[1] J.A. White, R. Klink, A. Alonso, A.R. Kay, J. Neurophysiol. 80, 262 (1998).

[2] J.A. White, J.T. Rubinstein, A.R. Kay, Trends Neurosci. 23, 131 (2000).

[3] B. Lindner, J. García-Ojalvo, A. Neiman, L. Schimansky-Geier, Phys. Rep. 392, 321 (2004).

[4] R. Jones, Nat. Rev. Neurosci. 6, 589 (2005).

[5] K. Wiesenfeld, F. Moss, Nature 373, 33 (1995).

[6] F. Moss, Biophys. J. 73, 2249 (1997).

[7] L. Gammaitoni, P. Hänggi, P. Jung, F. Marchesoni, Rev. Mod. Phys. 70, 223 (1998).

[8] G. Schmid, I. Goychuk, P. Hänggi, Europhys. Lett. 56, 22 (2001).

[9] P. Jung, J.W. Shuai, Europhys. Lett. 56, 29 (2001).

[10] G. Schmid, I. Goychuk, P. Hänggi, S. Zeng, P. Jung, Fluct. Noise Lett. 4, 33 (2004).

[11] L.H. Carney, Curr. Opin. Neurobiol. 9, 442 (1999).

[12] L.O. Trussell, Ann. Rev. Physiol. 61, 477 (1999).
[13] D. Oertel, Ann. Rev. Physiol. 61, 497 (1999).

[14] C.E. Carr, D. Soares, S. Parameshwaran, Curr. Opin. Neurobiol. 11, 727 (2001).

[15] E. Schneidman, B. Freedman, I. Segev, Neural. Comput. 10, 1679 (1998).

[16] A.D. Dorval, J. Neurosci. 25, 10025 (2005).

[17] A.L. Hodgkin, A.F. Huxley, J. Physiol. 117, 500 (1952).

[18] B. Hille, Ion Channels of Excitable Membranes, 3rd ed., Sinauer Associate, Inc., Sunderland, MA 2001.

[19] A. Manwani, C. Koch, Neural. Comput. 11, 1797 (1999).

[20] A.F. Strassberg, L.J. DeFelice, Neural. Comput. 5, 843 (1993).

[21] J.T. Rubinstein, Biophys. J. 68, 779 (1995).

[22] C.C. Chow, J.A. White, Biophys. J. 71, 3013 (1996).

[23] R.F. Fox, Biophys. J. 72, 2069 (1997).

[24] H. Mino, J.T. Rubinstein, J.A. White, Ann. Biomed. Eng. 30, 578 (2002).

[25] F. Reif, Fundamental of Statistical and Thermal Physics, McGraw-Hill, New York 1965. 